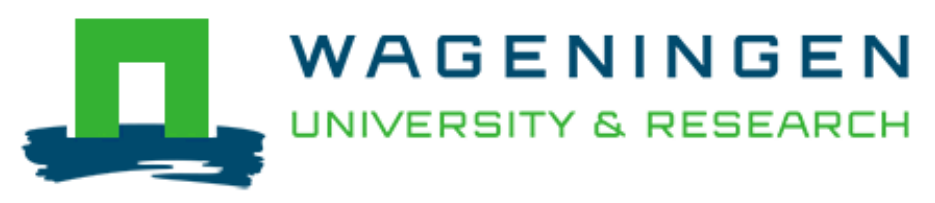

\author{
Anaerobic fungal communities differ along the horse digestive tract \\ Mura, E., Edwards, J., Kittelmann, S., Kaerger, K., Voigt, K., Mrázek, J., ... \\ Fliegerova, K.
}

This is a "Post-Print" accepted manuscript, which has been published in "Fungal Biology"

This version is distributed under a non-commercial no derivatives Creative Commons (c) (1) @) ( 9 (CC-BY-NC-ND) user license, which permits use, distribution, and reproduction in any medium, provided the original work is properly cited and not used for commercial purposes. Further, the restriction applies that if you remix, transform, or build upon the material, you may not distribute the modified material.

Please cite this publication as follows:

Mura, E., Edwards, J., Kittelmann, S., Kaerger, K., Voigt, K., Mrázek, J., ...

Fliegerova, K. (2019). Anaerobic fungal communities differ along the horse digestive tract. Fungal Biology, 123(3), 240-246.

https://doi.org/10.1016/j.funbio.2018.12.004 


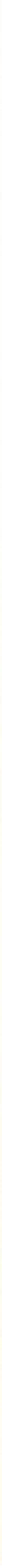




\title{
Anaerobic fungal communities differ along the horse digestive tract
}

\author{
Erica Mura ${ }^{1 \#, ~ J o a n ~ E d w a r d s ², ~ S a n d r a ~ K i t t e l m a n n ~}{ }^{3}$, Kerstin Kaerger ${ }^{4}, 5$, Kerstin Voigtt, ${ }^{4}$, \\ Jakub Mrázek $^{6}$, Giuseppe Moniello ${ }^{1}$ and Katerina Fliegerova ${ }^{6 *}$ \\ ${ }^{1}$ Department of Veterinary Medicine, University of Sassari, Via Vienna 2, 07100 Sassari,
} Italy

${ }^{2}$ Laboratory of Microbiology, Wageningen University \& Research, Wageningen, 6708 WE, The Netherlands

3 Wilmar International Ltd., Wil@NUS Corporate Lab, National University of Singapore, Singapore 117599, Singapore

${ }^{4}$ Institute of Microbiology, University of Jena, Neugasse 25, 07743 Jena, Germany

5 Leibniz Institute for Natural Product Research and Infection Biology, Jena Microbial Resource Collection, Adolf-Reichwein-Str. 23, 07745 Jena, Germany

${ }^{6}$ Institute of Animal Physiology and Genetics, CAS, Vídeňská 1083, Prague 14220, Czech Republic

\#All the authors contributed equally to this work.

*Corresponding author: Katerina Fliegerova. Institute of Animal Physiology and Genetics, Czech Academy of Sciences, Vídeňská 1083, Prague 14220, Czech Republic. Telephone: +420267090504. Fax: +420267090500. E-mail: fliegerova@iapg.cas.cz

\begin{abstract}
Anaerobic fungi are potent fibre degrading microbes in the equine hindgut, yet our understanding of their diversity and community structure is limited to date. In this preliminary work, using a clone library approach we studied the diversity of anaerobic fungi along six segments of the horse hindgut: caecum, right ventral colon (RVC), left ventral colon (LVC), left dorsal colon (LDC), right dorsal colon (RDC) and rectum. Of the 647 ITS1 clones, $61.7 \%$ were assigned to genus level groups that are so far without any cultured representatives, and $38.0 \%$ were assigned to the cultivated genera Neocallimastix $(35.1 \%)$, Orpinomyces (2.3\%), and Anaeromyces (0.6\%). AL1 dominated the group of uncultured anaerobic fungi, particularly in the RVC (88\%) and LDC (97\%). Sequences from the LSU clone library analysis of the LDC, however, split into two distinct phylogenetic clusters with
\end{abstract}


low sequence identity to Caecomyces sp. (94-96\%) and Liebetanzomyces sp. (92\%) respectively. Sequences belonging to cultured Neocallimastix spp. dominated in LVC (81\%) and rectum (75.5\%). Quantification of anaerobic fungi showed significantly higher concentrations in RVC and RDC compared to other segments, which influenced the interpretation of the changes in anaerobic fungal diveristy along the horse hindgut. These preliminary findings require further investigation.

Keywords: Anaerobic fungi; Diversity; Uncultured; ITS1; Horse; Hindgut

\section{Introduction}

Horses evolved as free-ranging herbivores of grassland environments, with an enlarged hindgut adaptation enabling them to obtain energy and nutrients from plant structural polysaccharides through microbial fermentation. The hindgut is comprised of two main fermentative chambers, the caecum and colon, which together constitute two-thirds of the volume of the digestive tract. The hindgut has a combined capacity of over $200 \mathrm{~L}$ (Frape 2010 ) and accounts for $75 \%$ of the mean transit time (23-48h) of dietary particles (van Weyenberg et al. 2006). The hindgut is home to bacteria, anaerobic fungi, methanogenic archaea and protozoa. Of these, the anaerobic fungi (Neocallimastigomycetes) are the most potent in terms of degrading plant fibres due to their complete and very efficient set of plant cell-wall degradation enzymes (Gruninger et al. 2014, Haitjema et al. 2014). Despite their presence in the horse hindgut within a few weeks of birth (Julliand et al. 1996), almost all the current knowledge of anaerobic fungi is derived from ruminant based studies.

Nine genera of anaerobic fungi validly described based on cultivated representatives, including those with filamentous monocentric (Neocallimastix, Piromyces, Oontomyces and Buwchfawromyces), filamentous polycentric (Orpinomyces, Anaeromyces and Pecoramyces) and bulbous (Caecomyces and Cyllamyces) mycelium (Gruninger et al. 2014, Edwards et al. 2017), have been recently extended by new monocentric genera Feramyces (Hanafy et al. 2018) and Liebetanzomyces (Joshi et al. 2018). These genera, however, represent only part of anaerobic fungal diversity as indicated by the large and growing numbers of internal transcribed spacer region 1 (ITS1) sequences in public databases that belong to potentially novel, as yet uncultured clades within the Neocallimastigomycetes. Based on the reevaluation of publicly available ITS1 sequences, which took into account both primary sequence and secondary structure information, Kittelmann et al. (2012) and later Koetschan 
et al. (2014) proposed a revised phylogeny and pragmatic taxonomy of anaerobic fungi, which resulted in 37 reproducible species or genus-level clades. Eighteen of these clades have not been cultured, containing only environmental derived sequences, and the number of not yet cultivated clades was recently increased to twenty-five (Paul et al. 2018).

A survey of anaerobic fungi in faeces of 30 different ruminant and non-ruminant herbivore species, found that members of the family Equidae clustered apart from the other nonEquidae herbivores studied (Liggenstoffer et al. 2010). The different domesticated and nondomesticated equines sampled in the Liggenstoffer et al. (2010) study shared a similar anaerobic fungal community, which was mainly composed of two novel genus level groups that are now termed AL1 \& AL3 (Koetschan et al. 2014). Cultivated anaerobic fungal genera were found in equine samples only in limited relative abundance (4-12\% of Caecomyces, $2 \%$ of Neocallimastix, $0.3 \%$ of Piromyces, $0.1-0.3 \%$ of Anaeromyces). These results suggest that the digestive tract of horses is largely occupied by novel, as yet uncultured anaerobic fungi, which differ from those previously described from foregut herbivores.

Whilst molecular based analysis of equine anaerobic fungal diversity has been performed on faecal samples (Liggenstoffer et al., 2010), it is known that bacterial, protozoal and archaeal community composition differs with hindgut segment (Julliand and Grimm 2016; Fliegerova et al. 2016). Therefore, there is a clear need to assess if the anaerobic fungal community composition also differs along the hindgut, particularly as niche differentiation within the anaerobic fungi has previously been proposed (Griffith et al. 2009). In this preliminary study, the anaerobic fungal community composition along the hindgut of a mature horse was determined using ITS1 based clone libraries.

\section{Materials and Methods}

\subsection{Collection of digesta samples from the horse hindgut}

Gut content samples were taken from the six segments of the hindgut of an Anglo-Arabian gelded male ( 24 years old) euthanised for non-research purposes in a local abattoir. The horse was maintained on a mixed diet of grass, meadow hay and complementary feed as described by Fliegerova et al. (2016), and was healthy with no history of any intestinal disorders. The segments sampled were: caecum, right ventral colon (RVC), left ventral colon (LVC), left dorsal colon (LDC), right dorsal colon (RDC) and rectum (representing faeces). Gut segments were tied off to prevent mixing between neighbouring segments, and the whole 
content from a section mixed thoroughly before a sample (approx $500 \mathrm{~g}$ ) was taken. Samples were placed in labelled plastic containers and transported on wet ice back to the laboratory where they were frozen $\left(\right.$ at $-20^{\circ} \mathrm{C}$ ) and then freeze-dried. Freeze-dried material was stored at $20^{\circ} \mathrm{C}$ until DNA extraction.

\subsection{DNA extraction}

Freeze-dried gut content (5 g) was homogenized using a mortar and pestle with liquid nitrogen. Genomic DNA was then extracted from $400 \mathrm{mg}$ of the resulting powder using the cetyltrimethylammonium bromide extraction protocol of Gardes and Bruns (1993) after modification with the respect to the amount of sample material used. The concentration and purity of extracted nucleic acids was checked using a NanoDrop 2000c UV-Vis spectrophotometer (Thermo Scientific, U.S.A), and DNA extracts were stored at $-20^{\circ} \mathrm{C}$ until use.

\subsection{PCR amplification}

Amplification of the anaerobic fungal ITS1 region from each sample was carried out with the combination of the fungal universal ITS1 forward primer (Gardes and Bruns 1993) and the Neocallimastigomycetes specific 5.8S rRNA gene reverse primer (Edwards et al. 2008). This resulted in an amplicon of approximately $350 \mathrm{bp}$ in length, as described previously by Fliegerova et al. (2010). The PCR reaction $(50 \mu \mathrm{l})$ was performed with a PPP Master Mix kit (Top-Bio, Czech Republic) and $0.3 \mu \mathrm{M}$ of each primer using the following thermal cycling conditions: 33 cycles of denaturation at $94^{\circ} \mathrm{C}$ for $1 \mathrm{~min}$, annealing at $58^{\circ} \mathrm{C}$ for $30 \mathrm{~s}$ and extension at $72^{\circ} \mathrm{C}$ for $45 \mathrm{~s}$ with an initial cycle of $94^{\circ} \mathrm{C}$ for $4 \mathrm{~min}$ and final cycle of $72^{\circ} \mathrm{C}$ for 2 min. Each sample was PCR amplified in quadruplicate and then pooled after successful amplification and verified by agarose gel electrophoresis.

For one sample, the LDC, the ribosomal large subunit 28S rRNA (LSU) was also amplified in quadruplicate with universal primers NL1 and NL4 as described previously by Fliegerova et al. (2006). The rationale for this analysis is explained in the results section.

Pooled PCR amplicons of the correct length were excised from an agarose gel with a sterile scalpel blade, purified and concentrated using a QIAquick Gel Extraction Kit (Qiagen, Germany).

\subsection{Construction of clone libraries and sequencing}


The TOPO ${ }^{\circledR}$ TA Cloning ${ }^{\circledR}$ Kit for Sequencing (Life Technologies, USA) was used for the preparation of the ITS1 clone libraries for each segment of equine hindgut, and the LSU clone library of the LDC segment. Ligation of the PCR amplicons into pCR4-TOPO vector was performed at room temperature for $30 \mathrm{~min}$ followed by transformation into competent One Shot TOP10 Escherichia coli cells (30 min on ice). Randomly selected clones of E. coli grown overnight in lysogeny broth plates with ampicillin $(50 \mu \mathrm{g} / \mathrm{ml})$ were checked by PCR with M13 primers for the presence of the ITS1 and LSU fragments of expected length. Plasmid DNA was then isolated from 881 ITS1 clones and 20 LSU clones using a GenElute $^{\mathrm{TM}}$ HP Plasmid Miniprep Kit (Sigma-Aldrich, USA). Purified plasmid DNAs were quantified using a NanoDrop ND-1000 UV-Vis Sectrophotometer (NanoDrop Technologies, USA) and Sanger sequenced (SEQme, Czech Republic). Generated sequences were checked for errors and any vector contamination removed. Of the clones sequenced, 647 of the ITS1 clones and 17 of the LSU clones yielded clear and unambiguous sequence data that were used for further analysis.

\subsection{Taxonomic and phylogenetic analysis}

Taxonomic assignment of ITS1 sequences (97\% identity threshold) was done in QIIME version 1.8 (Caporaso et al. 2010) by BLAST against the anaerobic fungal ITS1 reference database (Koetschan et al. 2014). The 647 cloned sequences with taxonomic assignment from the six libraries were concatenated into a single fasta file, and a sequence map (seqs_otus.txt) was manually built for input into the script "make otu table.py". The resulting relative abundance table was summarized at the clade level. A similar process was followed with the LSU sequence data, except the similarity search was carried out using BLAST against GenBank.

Evolutionary analyses of ITS1 sequences and the LSU sequences from the LDC sample were separately performed using the software MEGA 6 (Tamura et al. 2013) to construct phylogenetic trees, with 1,000 replicates for bootstrap analysis, using the UPGMA method. LSU genes of described genera Caecomyces (JQ782555, KM878679), Cyllamyces (DQ273829, KY386297), Piromyces (JF974096, JF974119, JN939159), Buwchfawromyces (KP205570, NG058679), Feramyces (MG584197, MG584228, MG605676), Neocallimastix (JF974094, JN939158, KT274174, KR920745), Pecoramyces (JN939127, KX961618), Orpinomyces (HQ703476, JN939163, KM878680), Anaeromyces (JN939157, JN939170, 
JN939172), Liebetanzomyces (MH468763) and Oontomyces (JX017314, JX017315) were used as reference sequences.

The ITS1 nucleotide sequences generated from the horse hindgut have been deposited in the GenBank database under the following accession numbers: MH038102 - MH038269 (caecum), MH038399 - MH038497 (RVC), MH038498 - MH038612 (LVC), MH038272 MH038398 (LDC), MH038613 - MH038694 (RDC) and MH038695 - MH038784 (rectum). The LSU sequences of the LDC have been deposited in the GenBank database under the accession numbers MH125212 - MH125228.

\section{6. qPCR analysis}

The MX 3005P QPCR System (Stratagene) and the Kapa SYBR Fast qPCR Master mix (Kapa Biosystems) was used for the qPCR determination of ITS1 gene copy numbers in each sample using the ITS1 primer pair and cycling conditions as described above (see PCR amplification). The standard curve was created (in triplicate) using a 10-fold dilution series of a pCR4-TOPO plasmid containing the ITS1 region of a Piromyces isolate (NCBI Accession No. KY368107). ITS1 copy numbers were determined (in triplicate) from $1 \mu 1$ of ten-fold diluted DNA, and data expressed per g of dry matter of digesta. Three replicates of a negative control sample without DNA template were also included in the qPCR assay. The qPCR assay efficiency was $101.5 \%$, and quantitation was linear over eight orders of magnitude $\left(10^{1}\right.$ to $10^{8}$ gene copy $\mu^{-1}$ ). The unpaired t-test (Microsoft Excel 2010) was used to identify significant differences in the counts of anaerobic fungi among all the samples. Significant differences were declared when $\mathrm{P}<0.05$. A quantitative profile of the anaerobic fungi was then calculated, using the ratio between the total ITS1 gene copy number and relative abundance of each clade for each hindgut segment.

\section{Results}

\subsection{ITS1 based diversity analysis of anaerobic fungi in the horse hindgut}

Of the 647 successfully sequenced ITS1 clones from all six segments of the horse hindgut, the 645 clones appeared to be anaerobic fungal in origin and only 2 of these sequences $(0.3 \%)$ could not be further taxonomically assigned within the class Neocallimastigomycetes using either the anaerobic fungal ITS1 reference database or GenBank. On average $61.7 \%$ of all the sequences were represented by genus-level groups that have so far only been described in the literature based on sequence data: AL1, AL7, DT1 and KF1 (as defined by Koetschan 
et al. 2014). The cultured genera Neocallimastix, Orpinomyces and Anaeromyces represented the remaining $38.0 \%$ sequences that could be taxonomically assigned. Phylogenetic relationships of ITS1 sequences of uncultured anaerobic fungi generated from the horse hindgut and cultured genera is shown in Fig. S1.

\subsection{Distribution and quantification of anaerobic fungi along the horse hindgut}

The relative abundance of the different anaerobic fungal clades (as defined by Koetschan et al. 2014) along the six segments of the horse hindgut are shown (Fig. 1.). The AL1 clade represented $53 \%$ of the total sequences and was present throughout the hindgut, however, its relative abundance differed among gut segments. AL1 represented almost all of the anaerobic fungi in the RVC (88\%) and LDC (97\%), and was the most abundant clade in the caecum $(62 \%)$ and RDC (53\%). In contrast, the relative abundance of AL1 in the LVC (4\%) and the rectum (3\%) was very low. Generally, the decreased abundance of AL1 in certain gut segments seemed to be associated with an increase in the abundance of the Neocallimastix 1 clade. This clade was the second most abundant (35.1\% of the total sequences) and was detected in all segments. The third most abundant clade was AL7 (4.3\% of the total sequences), and was also detected in all segments. In contrast, the KF1 clade $(4.2 \%$ of the total sequences) was only detected in the $\operatorname{LVC}(7 \%)$, RDC (3\%) and rectum $(17 \%)$. Sequences of the other less abundant fungi (Orpinomyces 1a, Orpinomyces 1b, Anaeromyces 1 and DT1) were relatively minor (13.7\% of total sequences) and detected only in some hindgut segments.

Total anaerobic fungal concentrations differed between segments, with the exception of the caecum and LDC (Fig. 2.). The RVC followed by RDC was found to contain the highest concentrations of anaerobic fungi, and the lowest concentrations of anaerobic fungi were detected in the LVC and rectum. It can be clearly seen that the lower concentrations of total anaerobic fungi in these segments is associated with a substantial decrease in the amount of the AL1 clade, rather than an increase in the Neocallimastix 1 clade (as indicated by Fig. 1.).

Fig. 1

Fig. 2

\subsection{LSU based diversity analysis of the LDC}

As $97 \%$ of the ITS1 sequences from the LDC segment belonged to the monophyletic AL1 clade (Fig. 3), this segment was also analysed using an LSU based clone library in order to 
better elucidate the taxonomic position of this uncultured clade. Tree topology showed that the LSU sequences obtained $(\mathrm{n}=17)$ formed two distinct clusters (Fig. 4). The clones representing cluster $I(n=8)$ had highest sequence identity $(94-96 \%)$ to a sequence belonging to a cultured Caecomyces communis (KM878679), and formed a sister group to the Caecomyces/Cyllamyces group. The clones representing cluster II $(n=9)$ had highest sequence identity (91 - 92\%) to a sequences belonging to a cultured Liebetanzomyces sp. (MH468763) and Anaeromyces sp. (MG605690), however, the cloned sequences clustered distantly from the reference anaerobic fungi present in the tree.

Fig. 3.

Fig. 4.

\section{Discussion}

Gut microbes are essential colonizers of the mammalian digestive tract. They are involved in food decomposition, production of vitamins and micronutrients. Microbial fermentative endproducts (especially acetate, propionate and butyrate) supply the host body by energy. Microbiota of digestive tract is also involved in a variety of other metabolic and physiological functions including the shaping of the immune system. The importance of the equine intestinal microbial ecosystem for animal health and performance is well established (Dicks et al. 2014), however, research has primarily focussed on only bacteria. The investigation of the anaerobic fungi in the equine hindgut to date has been limited and, to the best of our knowledge, there is no study of the diversity of anaerobic fungi in the different anatomophysiological segments of the horse hindgut.

Our work showed that an uncultured Neocallimastigales clade named AL1 was prevalent in the equine hindgut. This finding is in agreement with an ITS1 based next generation sequencing study that found the NG1 (=AL1 in Kittelmann et al. 2012) group could account for $56.7 \%-99.9 \%$ of the total anaerobic fungi in the faeces of certain horses and zebra (Liggenstoffer et al. 2010). It was surprising to observe that the AL1 clade was greatly decreased in the LVC and rectum, with the reason for these decreases not clear.

The AL1 clade is not specific to equines, as it was also detected (17.8-49.5\%) in a variety of other foregut fermenters (Liggenstoffer et al. 2010). However, ITS1 sequences of the AL1 clade retrieved in this study were most similar or identical only with those from the zebra and horse (deposited in the GenBank database). The effort to elucidate the phylogenetic 
relationships of the ITS1 defined AL1 clade using the LSU resulted in splitting of the sequences into two non-related clusters. As this contrasted the monophyletic clade seen with ITS1, further work is needed to confirm the basis of this finding.

The second most numerous clade of uncultivated anaerobic fungi identified in horse faeces by Liggenstoffer et al. (2010), clade NG3 (=AL3 in Kittelmann et al. 2012), has not been found in any part of the horse hindgut sampled in this study. On the other hand, sequences of the group NG7 (=AL7 in Kittelmann et al. 2012) retrieved by Liggenstoffer et al. (2010) in low relative abundance only from Somali wild ass faeces $(0.6 \%)$, represented $4 \%$ of total clones in the present study. Sequences of another group of anaerobic fungi, the KF1 clade, which represented $17 \%$ of the sequences in the horse rectum in this study, were first detected in cow manure and were described as an undistinguished Cyllamyces/Caecomyces cluster of uncultured fungi (Fliegerova et al. 2010).

Regarding the known cultivable anaerobic fungi, only the clade Neocallimastix 1 colonized the hindgut of the studied horse with a dominant relative abundance in the LVC and rectum. However, quantitative profiling showed that this was due to the absence of clade AL1, rather than an increased amount of Neocallimastix 1 in these segments. The Neocallimastix 1 sequences were mostly similar to those obtained from ruminants, especially from cow, bison and yak. Neocallimastix is known by its excellent hydrolytic properties and multi-functional cellulosomal enzymes, and is presumed to be a very effective (hemi)cellulose degrader (Gruninger et al. 2014, Wei et al. 2016). In horse, zebra and donkey faeces analysed by Liggenstoffer et al. (2010) this genus was either absent or represented at very low relative abundance $(<2 \%)$. However, in a sample from a Somali wild ass, Neocallimastix represented $45 \%$ of all sequences.

Whilst the cause of the considerable difference in anaerobic fungal diversity between the RVC and LVC is not clear, the difference between the neighbouring LVC and LDC may be related to the pelvic flexure. This narrow junction is responsible for the selective retention of coarse particles. Less digested particles are retained in the caecum and LVC, whereas liquid and finer particles move on into the LDC. These retropulsive-propulsive movements keep the caecum and the whole ventral colon filled, increasing the fermentation time of plant biomass. These segments of the hindgut are, therefore, the sites with the highest digesta mean retention time of (about 9 hours) and represents the main site of lignocellulose degradation (Van 
Weyenberg et al. 2006). As well as a longer time to degrade the structural polysaccharides, this also provides more time for the propagation of microorganisms.

The implication of the RVC ecosystem in forage degradation has been highlighted already by de Fombelle et al. (2003), due to the decreased proportion of cellulose in the digesta after passing through the RVC. This corresponds well with our finding of highest anaerobic fungal concentrations in the RVC compared to the other hindgut segments. On the other hand, the segments with the lowest anaerobic fungal concentrations (LVC and rectum) were associated with a decrease in the amount of the AL1 clade. Variations in the concentration of anaerobic fungi found among different gut segments in this study, however, contrasts with study of Dougal et al. (2012) where no effect of gut segment was seen based on the caecum, RDC and rectum sites that were sampled.

The RDC is the site of another selective mechanism (known as the colonic separation mechanism). This mechanism is responsible for the prolonged retention of fluid and smaller food particles (Drogoul et al. 2000). How this mechanism may contribute to the decrease of the AL1 clade in the rectum is not clear. Digesta samples from this part of the hindgut, could in future studies be separated into a liquid and a solid fraction to provide further insight, as anaerobic fungal vegetative biomass (as opposed to the motile zoospores) is tightly attached to plant particles.

Faeces samples are often used as reference samples to describe the microbial population of the digestive tract, because the faecal microbiota might be expected to contain representatives from all regions of the large intestine. Our study provides evidence that horse faecal samples can serve as qualitative reference samples, as almost all clades of anaerobic fungi found in the hindgut were also present in the faeces sample. The only exception was Anaeromyces, which was only detected in some segments and at very low relative abundances. However, the relative abundance and concentrations of anaerobic fungi differed considerably along the horse hindgut and the functional implications of this variation requires further investigation.

Differences in anaerobic fungal concentrations along the ruminant digestive tract is known to occur (Davies et al., 1993). Furthermore, a cultivation based study by Griffith et al. (2009) compared the anaerobic fungi in the rumen and faeces of the same cow, and found considerable differences in the abundance of the different taxa. Fungi with bulbous morphotypes (Caecomyces and Cyllamyces) were the most abundant genera in fresh faeces, where they comprised a 5-fold greater proportion of the total fungal population than in the 


\section{Acknowledgement}

This work was supported by the Ministry of Education, Youth and Sports of the Czech Republic, grant no. CZ.02.1.01/0.0/0.0/15_003/0000460 OP RDE) and programs of the University of Sassari Erasmus+, Master and Back 2014/2015 and Visiting Professor 2016/2017 Regione Autonoma Sardegna. JEE acknowledges funding from an EU H2020 Marie Curie Fellowship (706899).

\section{References}

Caporaso J.G., Kuczynski J., Stombaugh J., Bittinger K., Bushman F.D., Costello E.K., Fierer N., Pẽa A.G., Goodrich J.K., Gordon J.I., Huttley G.A., Kelley S.T., Knights D., Koenig J.E., Ley R.E., Lozupone C.A., McDonald D., Muegge B.D., Pirrung M., Reeder J., Sevinsky J.R., Turnbaugh P.J., Walters W.A., Widmann J., Yatsunenko T., Zaneveld J., Knight R., 2010. QIIME allows analysis of high-throughput community sequencing data. Nat. Methods 7, 335-336.

de Fombelle A., Varloud M., Goachet A.G., Jacotot E., Philippeau C., Drogoul C., Julliand V., 2003. Characterization of the microbial and biochemical profile of the different segments of the digestive tract in horses given two distinct diets. J. Anim. Sci. 77, 293-304.

Davies D.R., Theodorou M.K., Lawrence M.I.G., Trinci A.P.J., 1993. Distribution of anaerobic fungi in the digestive tract of cattle and their survival in feces. J. Gen. Microbiol. $139,1395-1400$. 
Dicks L.M.T., Botha M., Dicks E., Botes M., 2014. The equine gastro-intestinal tract: An overview of the microbiota, disease and treatment. Livest. Sci. 160, 69-81.

Dougal K., Harris P.A., Edwards A., Pachebat J.A., Blackmore T.M., Worgan H.J., Newbold C.J., 2012. A comparison of the microbiome and the metabolome of different regions of the equine hindgut. FEMS Microbiol. Ecol. 82, 642-652.

Drogoul C., Poncet C., Tisserand J.L., 2000. Feeding ground and pelleted hay rather than chopped hay to ponies: 1 . Consequences for in vivo digestibility and rate of passage of digesta. Anim. Feed. Sci. Technol. 87, 117-130.

Edwards J.E., Forster R.J., Callaghan T.M., Dollhofer V., Dagar S.S., Cheng Y., Chang J., Kittelmann S., Fliegerova K., Puniya A.K., Henske J.K., Gilmore S.P., O'Malley M.A., Griffith G.W., Smidt H., 2017. PCR and omics based techniques to study the diversity, ecology and biology of anaerobic fungi: insights, challenges and opportunities. Front. Microbiol. 8, 1657. DOI: 10.3389/fmicb.2017.01657

Edwards J.E., Kingston-Smith A.H., Jimenez H.R., Huws S.A., Skøt K.P., Griffith G.W., McEwan N.R., Theodorou M.K., 2008. Dynamics of initial colonization of nonconserved perennial ryegrass by anaerobic fungi in the bovine rumen. FEMS Microbiol. Ecol. 66, 537545.

Fliegerová, K., Mrázek, J., Voigt, K., 2006. Differentiation of anaerobic polycentric fungi by rDNA PCR-RFLP. Folia Microbiol. 51, 273-277.

Fliegerova K., Mrazek J., Hoffmann K., Zábranská J., Voigt K., 2010. Diversity of anaerobic fungi within cow manure determined by ITS1 analysis. Folia Microbiol. 55, 319-325.

Fliegerova K., Mura E., Mrazek J., Moniello G., 2016. A comparison of microbial profiles of different regions of the equine hindgut. Livest. Sci. 190, 16-19.

Frape, D. 2010. Equine Nutrition and feeding, fourth ed. Blackwell Publishing, Australia.

Gardes M., Bruns T.D., 1993. ITS primers with enhanced specificity for Basidiomycetes application to the identification of mycorrhizas and rusts. Mol. Ecol. 2, 113-118.

Griffith G.W, Ozkose E., Theodorou M.K., Davies D.R., 2009. Diversity of anaerobic fungal populations in cattle revealed by selective enrichment culture using different carbon sources. Fungal Ecol. 2, 87-97. 
Gruninger R.J., Anil K., Puniya A.K., Callaghan T.M., Edwards J.E., Youssef N., Dagar S.S., Fliegerova K., Griffith G.W., Forster R., Tsang A., McAllister T., Elshahed M.S., 2014. Anaerobic fungi (phylum Neocallimastigomycota): Advances in understanding of their taxonomy, life cycle, ecology, role, and biotechnological potential. FEMS Microbiol. Ecol. 90, 1-17.

Haitjema C.H., Solomon K.V., Henske J.K., Theodorou M.K., O'Malley M.A., 2014. Anaerobic gut fungi: Advances in isolation, culture, and cellulolytic enzyme discovery for biofuel production. Biotechnol. Bioeng. 111, 1471-1482.

Hanafy R.A., Elshahed M.S., Youssef N.H., 2018. Feramyces austinii, gen. nov., sp. nov., an anaerobic gut fungus from rumen and fecal samples of wild Barbary sheep and fallow deer. Mycologia 110, 513-525. https://doi.org/10.1080/00275514.2018.1466610

Jimenez H.R., Edwards J.E., McEwan N.R., Theodorou M.K., 2007. Characterisation of the population structure of anaerobic fungi in the ruminant digestive tract. Microb. Ecol. Health Dis. 19, 40.

Joshi A., Lanjekar V.B., Dhakephalkar P.K., Callaghan T.M., Griffith G.W., Dagar S.S., 2018. Liebetanzomyces polymorphus gen. et sp. nov., a new anaerobic fungus (Neocallimastigomycota) isolated from the rumen of a goat. Mycokeys 40, 89-110. https://doi.org/10.3897/mycokeys.40.28337

Julliand V., Grimm P., 2016. Horse species symposium: The microbiome of the horse hindgut: History and current knowledge. J. Anim. Sci. 94, 2262-2274. https://doi.org/10.2527/jas.2015-0198

Julliand V., De Vaux A., Villard L., Richard Y., 1996. Preliminary studies on the bacterial flora of faeces taken from foals, from birth to twelve weeks. Effect of the oral administration of a commercial colostrum replacer. Pferdeheilkunde 12, 209-212.

Koetschan C., Kittelmann S., Lu J., Al-Halbouni D., Jarvis G.N., Müller T., Wolf M., Janssen P.H., 2014. Internal transcribed spacer 1 secondary structure analysis reveals a common core throughout the anaerobic fungi (Neocallimastigomycota). PLoS ONE 9, e91928. https://doi.org/10.1371/journal.pone.0091928

Kittelmann S., Naylor G.E., Koolaard J.P., Janssen P.H., 2012. A proposed taxonomy of anaeobic fungi (class Neocallimastigomycetes) suitable for large-scale sequence-based 


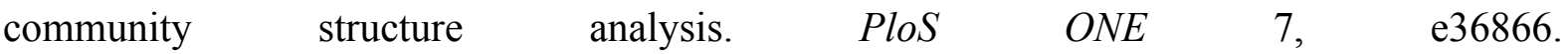
https://doi.org/10.1371/journal.pone.0036866

Liggenstoffer A.S., Youseff N.H., Couger M.B., Elshahed M.S., 2010. Phylogenetic diversity and community structure of anaerobic gut fungi (phylum Neocallimastigomycota) in ruminant and non-ruminant herbivores. ISME J. 4, 1225-1235.

Paul S.S., Bu D., Xu J., Hyde K.D., Yu Z., 2018. A phylogenetic census of global diversity of gut anaerobic fungi and a new taxonomic framework. Fungal Divers. 89, 253-266. https://doi.org/10.1007/s13225-018-0396-6

Tamura K., Stecher G., Peterson D., Filipski A., Kumar, S., 2013. MEGA6: Molecular Evolutionary Genetics Analysis version 6.0. Mol. Biol. Evol. 30, 2725-2729.

Van Weyenberg, S., Sales, J., and Janssens G.P.J., 2006. Passage rate of digesta through the equine gastrointestinal tract: A review. Livest. Sci. 99, 3-12.

Wei Y.Q., Yang H.J., Luan Y., Long R.J., Wu Y.J., Wang Z.Y., 2016. Isolation, identification and fibrolytic characteristics of rumen fungi grown with indigenous methanogen from yaks (Bos grunniens) grazing on the Qinghai-Tibetan Plateau. J. Appl. Microbiol. 120, 571-587.

\section{Figure captions}

Fig. 1. Relative abundances of anaerobic fungal clades (as defined by Koetschan et al. 2014) in the ITS1 clone libraries constructed from six segments of the horse hindgut. The total number of clones in each library is indicated in parentheses on the x-axis. The arrow indicates the pelvic flexure position between the LVC and LDC.

Fig. 2. Quantitative profiles of anaerobic fungal clades (as defined by Koetschan et al. 2014) in the ITS1 clone libraries constructed from six segments of the horse hindgut. The top of the bars indicate mean concentrations $(n=3)$ of total anaerobic fungal ITS1 gene copies in six different segments of horse hindgut, and different letters indicate significant differences (P $<0.05)$. Quantitative profiles were then generated from clade relative abundances and the 
ITS1 concentrations in each segment. The arrow indicates the pelvic flexure position between the LVC and LDC.

Fig. 3. Phylogenetic relationships of ITS1 sequences of anaerobic fungi generated from the LDC inferred using the UPGMA method with bootstrap values from 1,000 replications. The evolutionary distances were computed using the Jukes-Cantor method. The analysis involved a total of 142 nucleotide sequences (127 sequences generated from the LDC in this study and 14 of the most closely related sequences plus 1 outgroup sequence).

Fig. 4. Phylogenetic relationships of anaerobic fungal LSU gene sequences generated from the LDC inferred using the UPGMA method with bootstrap values from 1,000 replications. The evolutionary distances were computed using the Jukes-Cantor method. The analysis involved a total of 45 nucleotide sequences (17 sequences generated from the LDC, 27 sequences of the cultured genera of anaerobic fungi plus 1 outgroup sequence). 


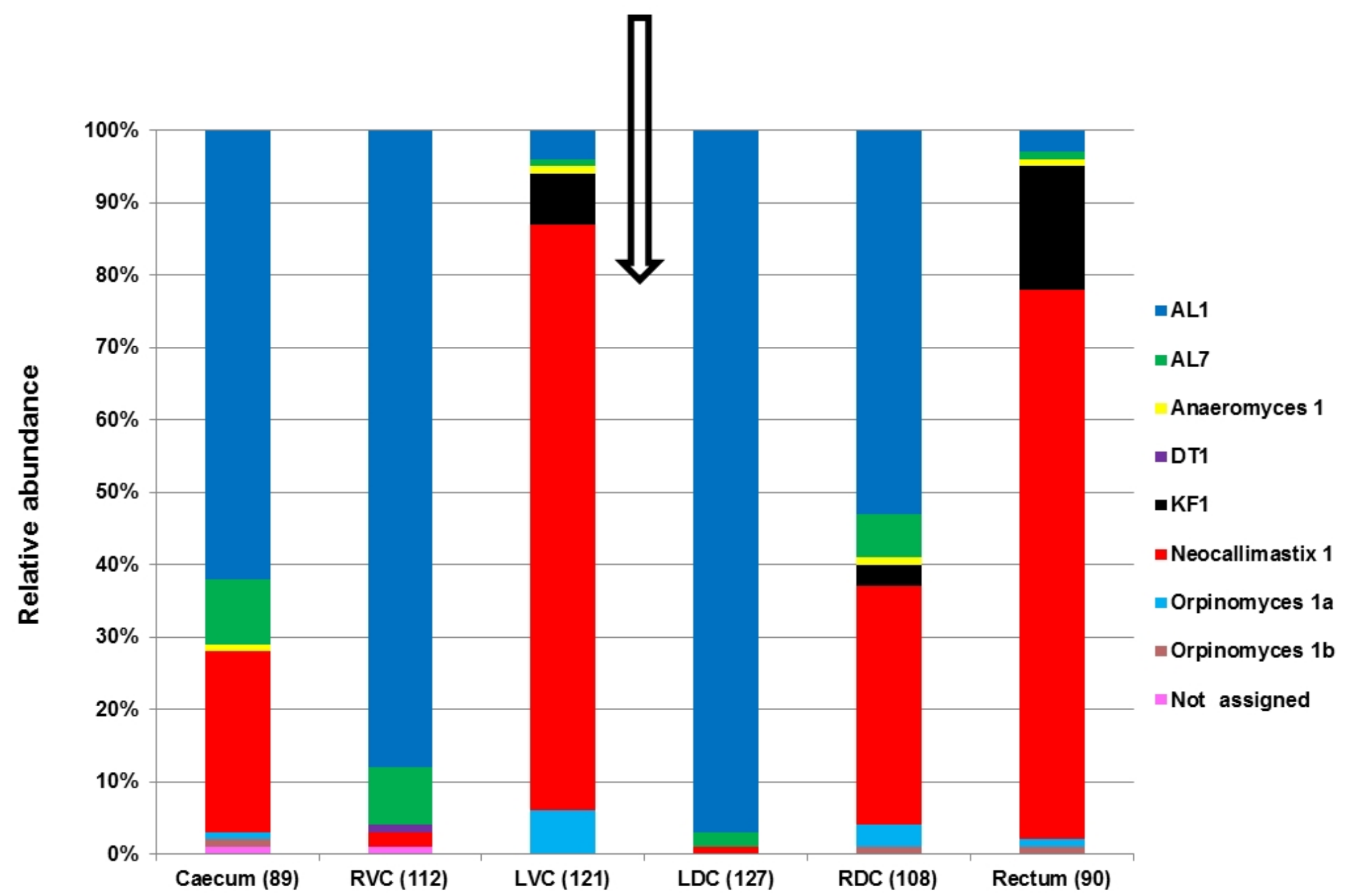




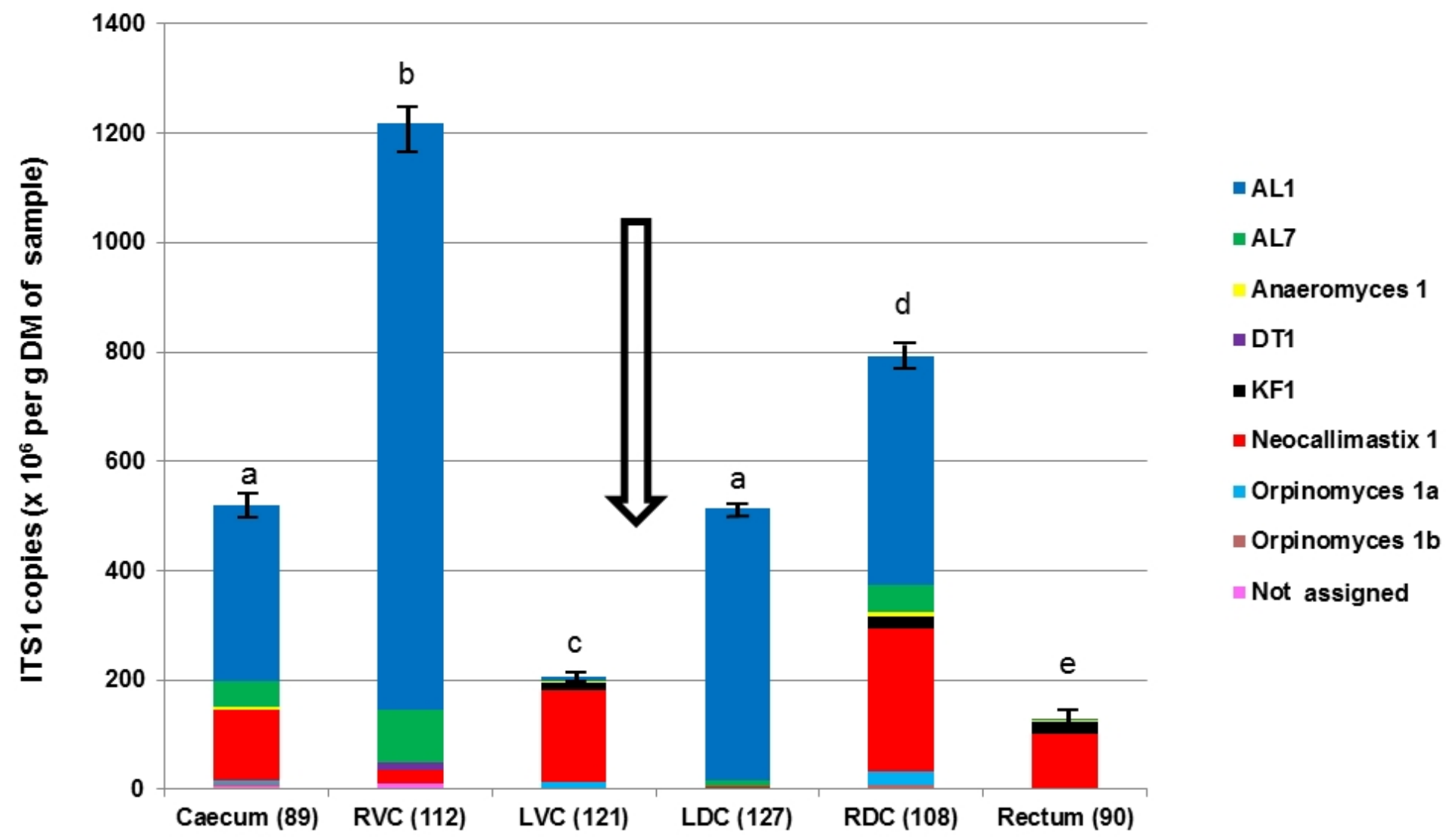


82 Uncultured Neocallimastigales clone WildAss01AOJA5 (GQ686305)

97 Caecomyces sp. W101 (DQ067604)

Caecomyces communis OF1 (KM878677)

60 100 LDC ITS1 seq175

- Uncultured fungus clone 1F1-41 (JX184439)

Neocallimastix 1

64 Anaeromyces sp. JB-1999 isolate AUC2 (AF170188)

100 Anaeromyces sp. AMG-2014 isolate AIB16 (KF789509)

Issatchenkia sp. YS5 (AM233510) 
